\title{
Filigrane
}

Écoutes psychothérapiques

\section{Psychanalyse et psychiatrie}

\section{Gilles Chagnon}

Volume 17, numéro 1, printemps 2008

L’avenir du clinicien I

URI : https://id.erudit.org/iderudit/018787ar

DOI : https://doi.org/10.7202/018787ar

Aller au sommaire du numéro

Éditeur(s)

Revue Santé mentale au Québec

ISSN

1192-1412 (imprimé)

1911-4656 (numérique)

Découvrir la revue

\section{Citer cet article}

Chagnon, G. (2008). Psychanalyse et psychiatrie. Filigrane, 17(1), 29-43.

https://doi.org/10.7202/018787ar

\section{Résumé de l'article}

La psychiatrie et la psychanalyse ont jadis cohabité dans une sorte de complémentarité. À l'ère des données probantes et de l'« evidence based medecine », la psychanalyse se voit maintenant accusée d'être incapable d'offrir des résultats validés scientifiquement. Ses avancées cliniques, sa métapsychologie sont devenues suspectes. Pourtant, compte tenu de la complexité de l'appareil psychique, il demeure vital d'utiliser une pluralité de modalités épistémologiques pour dynamiser les voies de recherche, tant sur le plan théorique que clinique. Comment, et à quelles conditions, poursuivre le dialogue toujours nécessaire entre psychiatrie et psychanalyse ?
Ce document est protégé par la loi sur le droit d'auteur. L’utilisation des services d'Érudit (y compris la reproduction) est assujettie à sa politique d'utilisation que vous pouvez consulter en ligne.

https://apropos.erudit.org/fr/usagers/politique-dutilisation/ 


\title{
Psychanalyse et psychiatrie
}

gilles chagnon

\begin{abstract}
La psychiatrie et la psychanalyse ont jadis cohabité dans une sorte de complémentarité. À l'ère des données probantes et de l'«evidence based medecine», la psychanalyse se voit maintenant accusée d'être incapable d'offrir des résultats validés scientifiquement. Ses avancées cliniques, sa métapsychologie sont devenues suspectes. Pourtant, compte tenu de la complexité de l'appareil psychique, il demeure vital d'utiliser une pluralité de modalités épistémologiques pour dynamiser les voies de recherche, tant sur le plan théorique que clinique. Comment, et à quelles conditions, poursuivre le dialogue toujours nécessaire entre psychiatrie et psychanalyse?
\end{abstract}

«La différence n'est pas ce qui masque ou édulcore le conflit: elle se conquiert sur le conflit, elle est au-delà et à côté de lui.»

Roland Barthes, Le plaisir du texte

\section{L'état des choses}

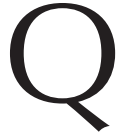

uand on songe aux rapports actuels entre la psychiatrie et la psychanalyse, on se trouve apparemment dans un lieu conflictuel et inquiet de la pensée. En quête de sa légitimité médicale et scientifique, la psychiatrie au cours des deux dernières décennies a, de façon accélérée, déclassé de son corpus le savoir et la pratique psychanalytiques. Signe des temps : même le vocable «psychanalyse» est mis à mal; on lui préfère son double édulcoré, la psychodynamique. Pourtant l'histoire de la psychiatrie, depuis son avènement autour de l'époque de Philippe Pinel, a été marquée de constants allers-retours entre les postulats organicistes et spiritualistes qui cherchaient à théoriser l'appareil psychique.

Essentiellement, la psychanalyse serait invalidée aujourd'hui sur le fait que ses bases théoriques ne sont pas scientifiques et qu'elle n'a pas d'efficacité prouvée sur le plan clinique. Si on veut bien lui concéder une efficacité, c'est pour s'empresser de préciser que c'est une efficacité qui ne lui est pas spécifique mais qui relève plutôt des ingrédients propres à toute forme de psychothérapie, quelle qu'en soit l'allégeance théorique.

C'est pourquoi aujourd'hui, dans les facultés de médecine, on apprend très tôt aux futurs médecins que la psychanalyse est un savoir non scientifique, donc qu'il n'y a plus lieu de l'inclure comme un savoir validé dans les théories du psychisme. Dans la formation du futur psychiatre, les théories et la pratique «psychodynamiques» sont encore présentées mais elles ont été mises au pas et ne sont devenues qu'une des modalités de la rubrique psycho du bio-psycho-social. Par exemple, dans les départements de psychiatrie des universités francophones 
québécoises, une place est encore gardée à l'apprentissage de la psychothérapie dynamique (brève ou à long terme) chez les futurs psychiatres. Les résidents y apprennent donc à manier certains éléments de cette écoute non directive basée sur la libre association et le repérage du transfert. Ce faisant, ils sont exposés à l'utilisation clinique des notions de base qui leur ont été enseignées concernant les conflictualités inconscientes, les mécanismes de défense, l'étayage pulsionnel, les stades de la libido, etc. Mais le statut de cette forme d'enseignement demeure tout de même fragile face aux autres formes de psychothérapies «evidence based» dont l'efficacité clinique aurait, quant à elle, été démontrée hors de tout doute. On fait référence alors aux thérapies manuélisées que sont la TCC (thérapie cognitivocomportementale) et la thérapie interpersonnelle.

Les raisons de cette désaffection sont nombreuses. Sans prétendre à l'exhaustivité, j'en évoquerai ici quelques-unes.

Il est, premièrement, évident que les progrès récents de la neurobiologie et que les méthodes d'investigation sans cesse plus poussées du fonctionnement cérébral ont permis de faire des avancées remarquables sur le plan de la neurophysiologie, non seulement des pathologies mentales, mais également des processus cognitifs et des comportements humains dans leur ensemble. Ces progrès ont également eu des effets sur la dimension curative des affections psychiatriques. L'arsenal des traitements pharmacologiques a été renouvelé au cours des années quatre-vingtdix et continue de l'être. Les médicaments introduits aux cours des années cinquante et soixante comportaient une multitude d'effets secondaires qui nuisaient à la qualité de vie des patients; la nouvelle génération de psychotropes, mieux tolérée, propose donc des solutions beaucoup moins rédhibitoires aux individus souffrants. Conséquemment, l'usage de ces psychotropes s'est généralisé et ses indications se sont élargies. On ne peut que se réjouir de ces avancées nouvelles qui vont de pair avec les progrès des autres disciplines médicales. En contrepartie, un usage exclusif de ces solutions pharmacologiques peut contribuer à réifier la souffrance mentale et à déposséder l'individu du sens de sa souffrance. Si l'outil pharmacologique, dans son extériorité, prétend invalider la nécessité de l'élaboration de la souffrance psychique, si les avancées neurobiologiques s'imposent comme seules valides pour expliciter les aléas de la vie mentale, alors on se rapproche effectivement d'une robotisation de l'existence humaine.

Par ailleurs, deuxième raison, la fracture qui s'est produite entre le DSM II et le DSM III, publié en 1980, a conduit à l'expulsion du concept de névrose. La nosographie psychiatrique venait ainsi de se séparer officiellement de ses liens avec la psychanalyse. Les raisons invoquées dans l'introduction du DSM III concernaient la nécessité de mettre au point une nosographie qui se voulait a-théorique, c'est-à-dire dégagée de toute prétention quant à l'étiologie des affections mentales. Les principales psycho-névroses (obsessionnelle, phobique, hystérique) avaient été explorées abondamment par l'investigation psychanalytique depuis ses débuts et constituaient le fer de lance de la cure analytique. Avec l'éviction de ce concept de névrose, c'est non seulement un lien étiologique qui 
était rejeté, mais aussi, ce qui est beaucoup plus grave, la notion même de structure intra-psychique, c'est-à-dire les trois grandes structures invoquées alors: névrose, psychose et perversion. Le diagnostic multi-axial avec notamment le recours à l'axe II, la personnalité sous-jacente, n'a compensé que partiellement cette perte du référent structural. La question demeure entière: est-il vraiment possible de proposer un outil de classification qui soit vraiment purement descriptif et dénué de toute prémisse conceptuelle ou idéologique ? Est-ce qu'une entreprise phénoménologique peut être séparée du contexte historique et épistémologique qui conditionne son regard? À regarder de plus près la suite de l'histoire de la psychiatrie après la rupture introduite par le DSM III, on peut sérieusement en douter.

De plus, troisième raison, du côté de la psychanalyse, la nature très particulière de son objet d'étude, l'inconscient, et son rôle dans la vie intra-psychique, n'est sans doute pas étrangère à la fragmentation de son savoir en des écoles et des courants parfois très divergents. Ces divergences ne sont pas forcément néfastes; bien au contraire, elles témoignent de la complexité et de la vitalité de l'appareil théorique mis de l'avant par Freud et ses successeurs. En ce sens, il n'y aurait pas quelque chose comme «la» psychanalyse, au sens où on dirait «la» psychiatrie, faisant référence alors à un savoir validé par une institution, en l'occurrence le Collège royal des médecins du Canada qui, d'un océan à l'autre, certifie les futurs psychiatres. Au Québec, toutefois, cette pluralité des courants psychanalytiques n'a pas vraiment trouvé à s'exprimer ; conséquemment, la transmission de la psychanalyse s'est faite de façon souvent très dogmatique, voire même figée. Comment expliquer que tant de psychiatres psychanalystes aient eu des postes de commande au sein des institutions universitaires ou dans les établissements de santé jusqu'au seuil des années 90 et qu'il y ait eu, malgré cela, une désaffection massive des psychiatres envers la pratique psychanalytique ? Cette interrogation conduit à une question plus générale : qu'est-ce qui a cloché dans la transmission de la psychanalyse pour qu'elle se trouve si rapidement déclassée de l'univers théorique et clinique de la psychiatrie?

Enfin, quatrième raison, l'explosion de la demande de soins en santé mentale a accentué la nécessité de proposer des traitements facilement accessibles et de courte durée. Les grands enjeux de la santé sont devenus éminemment politiques, qui en douterait aujourd'hui, et ce fait amène sur le terrain une intrusion gestionnaire qui fait souvent fi des problèmes cliniques réels. L'espace de pensée que requiert la complexité de la clinique psychiatrique est souvent perçu comme une perte de temps et d'efficacité et, sur ce plan, la psychanalyse est incriminée de façon caricaturale. Comment ne pas voir que cette obsession d'une pratique efficace nous amène à parler davantage de concordance statistique plutôt que de la sémiologie toujours changeante de la folie moderne? Comment penser les liens entre la folie presque entièrement médicalisée d'aujourd'hui et les changements plus généraux (redéfinition des rôles familiaux, explosion du monde virtuel, métissage des cultures, etc.) survenus au niveau social qui affectent l'individu dans son rapport à lui-même et aux autres? Enfin, comment questionner les présupposés 
idéologiques, scientifiques, voire économiques, qui structurent dans l'ombre nos pratiques?

\section{La vérité statufiée}

L'évidence. Qui ne désire être éclairé par la lumière de l'évidence?

La Vérité existerait donc au bout de notre désir industrieux de savoir, entière, majuscule, radieuse, et surtout prête à orienter nos praxis qui, de se colleter jour après jour aux obscurités troublantes de la souffrance psychique, aspirent à l'évidence comme des plantes aspirent au soleil.

Ici malheureusement j'ironise à peine. Dans la plupart des assemblées de spécialistes, on fait référence sur un ton révérencieux au label «evidence based» pour désigner un processus scientifique de recherche de la vérité qui tend à s'imposer comme étant le seul valable. On est à l'ère de la médecine des données probantes, et la psychiatrie n'y échappe pas, même si elle risque d'y perdre son âme ${ }^{1}$. Les interventions proposées pour chacune des grandes catégories diagnostiques tendent à se retrouver dans des guides de pratique ou «guidelines». La validité de ces interventions est classée selon son niveau d'évidence (on compte trois niveaux dans cette classification), c'est-à-dire selon le nombre d'études qui ont été conduites sous le primat du «contrôlé randomisé» et qui ont confirmé l'efficacité de ce qui était ainsi mesuré. Nous nous trouvons ici dans le déploiement du visible et du mesurable.

Or, cette sériation des paramètres en vue de les mesurer (par exemple les paramètres de la dépression, du trouble borderline) suppose une réduction majeure du substrat phénoménologique à l'étude, à savoir le psychisme des sujets. Les échelles utilisées pour standardiser les études proposent une découpe de la réalité psychique qui frappe par son simplisme. S'il s'agit d'étudier l'efficacité d'un médicament, cette méthode expérimentale a toute sa place, comme ce fut d'ailleurs le cas bien avant l'arrivée du label «evidence based». Par contre, si cette méthode prétend valider ou invalider l'ensemble de ce qui compose la vie psychique, alors on entre dans un réductionnisme qui ne peut que faire violence à la recherche de vérité.

«Il est dorénavant devenu commun de penser et de concevoir une démarche scientifique et ce qu'elle exige de rigueur et de développement logique en la confondant avec une certaine méthode de recherche, le plus souvent la méthode expérimentale empruntée aux sciences exactes, dont l'application dans la recherche clinique par exemple garantirait la validité et l'objectivité de l'approche. Comme si une recherche clinique en psychiatrie ou en santé mentale, pour être reconnue «scientifique et rigoureuse», ne pouvait que suivre cette voie expérimentale et reposer sur des conditions, critères et données nécessairement «objectifs » parce que quantifiables. » (Cantin, 2005) 
C'est souvent au nom de cette méthode qu'on a voulu invalider les apports de la psychanalyse. Qu'on pense ici au fameux pamphlet Le livre noir de la psychanalyse paru en France en 2005, ou mieux encore aux PORT (Patient Outcome Research Team) Treatments Recommendations à propos de la schizophrénie. Cette recherche, au nom de l'evidence based psychiatry en est venue à statuer que les interventions psychodynamiques pouvaient être nuisibles dans l'arsenal des traitements de la schizophrénie ${ }^{2}$.

Sans vouloir dramatiser ce débat, il n'en reste pas moins qu'il y a, au sein d'une certaine psychiatrie de plus en plus dominante, une volonté souvent très peu voilée d'en «finir avec» l'héritage conceptuel et thérapeutique mis de l'avant par la psychanalyse pour approcher le réel, sous prétexte que l'efficacité de la psychanalyse n'est pas validée par la méthode expérimentale.

Cette prétention hégémonique mérite d'être dénoncée pour plusieurs raisons.

D'abord, parce qu'elle semble avoir oublié que l'objet d'étude de la psychiatrie n'est pas identique à celui des neurologues, et que l'étude du système psychique n'est pas superposable à l'étude des processus cérébraux, si loin que puissent nous amener les avancées de la neurobiologie.

Ensuite, parce que cette volonté totalisante contredit la pensée scientifique ellemême, qui doit pouvoir penser les limites de son champ d'application, faute de quoi elle est effectivement suspecte de «scientisme», c'est-à-dire d'une récupération idéologique de ses fondements. «Le scientisme défigure ce qu'il y a de plus respectable dans la science. [...] C'est donc bien au nom de la science qu'il faut être vigilant contre le scientisme et contre le positivisme scientiste.» (Derrida, 2001)

Enfin, parce que, malgré l'intensification des recherches au niveau génétique et neurophysiologique, la question de l'étiologie de la plupart des maladies mentales n'a toujours pas été élucidée, et qu'on continue à se référer à une causalité multifactorielle. Cette complexité multifactorielle exige en retour une pluralité de méthodes d'investigation.

Pour répondre à ses détracteurs, certains psychanalystes se sont efforcés de s'aligner sur ces critères de scientificité pour atteindre à une forme de légitimité. On veut faire la démonstration que la thérapie analytique peut figurer au rang des thérapies «evidence based». C'est en effet une avenue possible, qui semble déjà avoir colligé un certain nombre de données probantes. Mais il importe à mon avis de mener le débat au niveau où il doit se situer, c'est-à-dire dans ses fondements épistémologiques.

\section{D'une différence fondamentale}

Pour introduire la question épistémologique, j'aimerais d'abord articuler mon propos autour de ce qui constitue une différence fondamentale entre la psychiatrie et la psychanalyse dans le rapport au savoir.

Le psychiatre, en tant que spécialiste, doit se poser comme celui qui sait. Il est mandaté par le grand ensemble social à une fonction précise, qui est celle non 
seulement de soigner et de guider les malades mentaux dans leur évolution, mais aussi de gérer ou à tout le moins de superviser la place de la folie dans le tissu social. Il doit donc maîtriser le mieux possible le champ d'expertise de sa discipline, laquelle tend à s'uniformiser pour que tous les patients aient droit à des traitements de pointe reconnus comme efficaces. C'est ainsi que les guides de pratique ont vu le jour, proposant des algorithmes de traitement qui sont autant de points de repères en vue d'une bonne pratique. Il est important de prendre en compte que le processus mis en place par le Collège royal du Canada pour la certification des futurs psychiatres s'applique au Québec comme dans les autres provinces et qu'il n'est pas si différent du modèle américain. Au fil d'arrivée, le psychiatre doit posséder un savoir bien défini, uniformisé, ce qui à l'évidence oriente et encadre sa façon d'écouter, sa manière d'être avec le patient.

De plus, dans l'interface entre la psychiatrie et la loi, que ce soit au niveau civil ou criminel, allant de la rédaction des rapports d'invalidité à l'employeur à ceux statuant devant magistrat de la non-responsabilité criminelle de tel ou tel individu, il doit pouvoir sortir du huis clos de la rencontre et témoigner du lieu de son expertise particulière. Sans parler du rapport de force qui s'instaure dès qu'il y a en jeu une dangerosité auto ou hétéro-agressive chez un patient refusant de se faire soigner. C'est dire que le savoir dans ce contexte mène souvent à une action, qu'on pense seulement ici à l'acte de prescrire.

Dans l'intervention psychanalytique, la relation entre le savoir et la pratique est d'une certaine manière plus complexe. Il ne s'agit pas d'apporter au patient un savoir intellectuel sur sa problématique intra-psychique mais bien de descendre avec lui dans les méandres de ses mécanismes internes, et particulièrement dans ce qui résiste au mouvement des pulsions de vie. Alors que l'intervention psychiatrique doit être pensée comme l'examen d'une pluralité de signes et symptômes préalablement définis, examen devant mener le patient à entrer dans une catégorie diagnostique d'où découlera la logique des solutions thérapeutiques, le dispositif psychanalytique s'appuie quant à lui sur l'unicité de ce qui se met en place entre le patient et l'analyste, laquelle unicité se déploie entièrement dans le champ de la parole et du langage, avec en perspective la masse compacte de tout ce qui fait silence, même sur un plan transgénérationnel.

De plus, il ne faut pas oublier que, pour se trouver dans la position analytique, chaque analyste doit avoir fait l'épreuve, comme analysant, du dispositif analytique et de ses possibilités de transformation de la vie psychique. À travers l'analyse du transfert et sa dissolution éventuelle menant à la fin de l'analyse, le futur analyste aura donc éprouvé tout le tranchant de l'expression qui le rend «sujet supposé savoir» face à un autre en position de demande. C'est donc dire comment le rapport au savoir de l'analyste se fonde d'abord sur la singularité de sa propre démarche analytique. Bien sûr, devenir analyste suppose également avoir souscrit à un édifice de savoir qui a aussi ses notions fondamentales, son histoire, son lexique, ses divergences, de même qu'à des règles techniques qui vont guider son écoute et son travail interprétatif. Le travail du psychanalyste sera donc constitué 
d'un aller-retour constant entre le huis-clos de la relation thérapeutique et l'ensemble de son savoir qui, à travers le temps, lui aura permis d'édifier ses propres théories de la vie intra-psychique.

Donc, le «sujet supposé savoir» sait en effet quelque chose, mais ce savoir est, si l'on peut dire, constamment travaillé par la prise en compte de sa propre limite. À leur façon, Freud, Lacan et Bion notamment ont tous réfléchi sur l'importance d'une position de non-savoir à travers laquelle le clinicien analyste doit se laisser heurter ou rejoindre par les signifiants qui ordonnent la mise en scène de la vie psychique de celui qui vient lui parler. Le savoir est ici relativisé par tout ce qui lui résiste, et c'est bien à partir de ce qui s'oppose à sa compréhension et à son interprétation que l'analyste fera avancer les choses sur les plans clinique et théorique. C'est là une des manifestations majeures du travail de l'inconscient.

Il reste que le travail du psychiatre repose aussi en bonne partie sur sa capacité d'entrer en relation avec l'intimité du patient, à aller au-delà de la compréhension symptomatique pour apercevoir la personne dans la complexité de sa souffrance et de son histoire. Sans quoi il est voué à ne reproduire que des rencontres manquées et à se faire l'agent d'une machine à dispenser des soins qui, sous l'obsession de son efficacité, participe à un processus de désubjectivation et d'uniformisation qui est un des enjeux les plus inquiétants de la mondialisation. Chaque psychiatre doit en lui-même faire travailler le paradoxe entre son rôle d'expert dans un système de santé où le patient est un objet de soins et son souci d'une intervention qui puisse aussi interpeller l'autre en tant que sujet. Car c'est seulement dans la perspective d'une telle interpellation que la rencontre prendra une valeur pour le patient et qu'elle pourra devenir éventuellement source de changement psychique.

\section{La lettre contre le chiffre}

Si je tente de résumer en une formule dichotomique simple ce qui transparaît à la racine de cette différence épistémologique entre la psychanalyse et la méthode scientifique expérimentale, je dirais qu'il s'agit d'apercevoir le pouvoir de la lettre contre celui du chiffre.

Actuellement, tout rapport à la vérité en psychiatrie, toute validation de données nouvelles, semble devoir en passer par la puissance du chiffre. Les vérités evidence based ont un niveau d'évidence d'autant plus élevé qu'elles reposent sur des cohortes populeuses et sur un grand nombre d'études convergentes. De même, sur le plan de la gestion des soins, toute intervention doit pouvoir attester de son efficacité, dans la mesure où elle sera passée par une démarche évaluative reposant sur des aspects quantitatifs (l'idéal étant évidemment de traiter le plus grand nombre de patients dans le plus court laps de temps). Tout se passe donc comme si la validation de la signification devait nécessairement s'appuyer sur du quantifiable et du mesurable.

Qu'on comprenne bien ce que je cherche à poser ici : il ne s'agit pas d'invalider le pouvoir du chiffre et la pertinence du mesurable; la question des méta-analyses et la convergence des résultats vers des données probantes constitue un acquis 
important dans l'établissement de méthodes de traitement valides et applicables au plus grand nombre. Toutefois, il est urgent de penser les limites inhérentes à cette découpe du phénoménologique, de faire la déconstruction de ses présupposés idéologiques et économiques et d'apercevoir les enjeux qu'elle laisse dans l'ombre.

La psychanalyse, quant à elle, pourrait être définie comme la science du sujet. Qu'est-ce qui compose structuralement la subjectivité (point de vue topique), comment ces composantes s'articulent-elles entre elles (point de vue dynamique), et selon quels vecteurs d'intensité (point de vue économique)?

Or, s'il y a bien une spécificité du savoir psychanalytique, c'est de s'employer à élucider comment l'inconscient demeure prépondérant dans les manifestations de la vie psychique et dans ses aléas psychopathologiques. Cette notion d'inconscient ne réfère pas à une mystique des profondeurs, mais désigne ici une logique qui ordonne de façon sous-jacente le rapport du sujet à son désir, dans la mesure où ledit désir excède constamment chez lui son intentionnalité consciente. Que le je ne soit pas maître chez lui, c'est là une vérité scandaleuse qui a toujours procuré nombre de détracteurs à la psychanalyse depuis sa fondation.

Et ce n'est pas le moindre mérite de Lacan d'avoir articulé que cette logique de l'inconscient s'instaure chez le sujet à partir de la nécessité de sa venue au monde dans l'univers symbolique. C'est-à-dire que l'individu ne parvient à l'existence subjective qu'à travers l'assimilation d'un système signifiant qui lui préexiste, assimilation par laquelle il pourra dire je et s'inscrire dans la suite des générations. Ce qu'il s'agit d'apercevoir ici, c'est l'antériorité de la lettre sur le chiffre, et non seulement sur le chiffre mais sur l'avènement du sujet lui-même. Ou : l'inscription de la lettre comme préalable à la structuration de la réalité subjective.

On sait bien que le langage ne doit pas être considéré comme un simple véhicule de la pensée. Comme le dit Kristeva (1981), le langage est le matériau même de la pensée; dans la mesure où «il n'y a pas de pensée extra-linguistique ». À travers le langage, l'individu est aux prises avec une altérité qui l'empêche de coïncider tout à fait avec lui-même; le langage insère l'individu dans le lien social, dans l'histoire, et l'aliène du même coup.

Comme les topiques freudiennes, chacune à leur manière, nous permettent de modéliser quelque chose de la structure psychique, les trois registres Réel/ Symbolique/Imaginaire nous permettent d'aller au-delà de l'intersubjectivité (le moi et ses objets) qui se trouve prise au niveau de l'imaginaire. Le registre symbolique, notamment, permet de penser l'articulation du sujet avec un ordre langagier qui le conditionne - c'est le fameux grand Autre, qui n'est en rien réductible à la série des petits autres qui vont peupler le carrousel des désirs et des fantasmes de l'individu. Si, comme nous invite à le penser François Peraldi (2006), la psychanalyse introduit dans l'horizon du savoir une "coupure épistémologique», c'est dans la mesure où le savoir qu'elle institue a partie liée avec le pouvoir singulier du signifiant, dans toute sa dimension polysémique.

Malgré les nombreuses prédictions annonçant la fin de la psychanalyse, il n'en reste pas moins qu'elle demeure toujours en ce début du $\mathrm{XXI}^{\mathrm{e}}$ siècle un lieu 
d'élaboration extraordinairement prolifique. La psychanalyse continue d'apporter des éclairages précieux sur la clinique moderne et aussi à travers ses applications multiples, que ce soit en relation avec d'autres sciences humaines, avec les études littéraires, la philosophie, etc. Son corpus s'est complexifié et diversifié à travers plus d'un siècle de recherche. Il serait dommage et inquiétant que la psychiatrie se piège dans une méthode unique de recherche de la vérité et délaisse d'autres modes épistémiques qui demeurent plus que jamais nécessaires pour rendre compte de la complexité du système psychique.

Le véritable enjeu de la psychiatrie actuelle, sa difficulté, son défi, est de dynamiser le dialogue entre les divers courants qui alimentent son savoir, d'endurer les zones d'ombre qui sont constitutives de tout processus de mise en langage du phénoménal. Beaucoup d'auteurs tentent de réinstaurer ce dialogue entre les données nouvelles de la recherche scientifique et les développements issus de la spéculation métapsychologique de la psychanalyse, et les avenues ne manquent pas pour relancer l'essor de la collaboration entre la psychiatrie et la psychanalyse. J'en aborderai quelques-unes en cernant d'un peu plus près le travail que j'effectue depuis bientôt dix ans auprès des psychotiques.

\section{Par exemple, la psychose}

Je travaille depuis quelques années dans un hôpital général en tant que psychiatre responsable de l'hôpital de jour, Le Point de Prise, s'employant au traitement de patients psychotiques. La composition même de l'équipe interdisciplinaire de l'hôpital de jour traduit que les interventions faites auprès des patients sont de nature bio-psycho-sociales ${ }^{3}$. La complexité des situations cliniques rencontrées exige un éclectisme des approches. En plus du suivi individuel assuré par le psychiatre et par son intervenante principale, chaque patient assiste à des rencontres en petits groupes consistant en des ateliers d'ergothérapie, de psycho-éducation, de travail sur la cognition, l'intégration corporelle, etc. Certains patients, parfois reclus depuis des mois, voire des années, viennent y renouer du lien avec autrui. Un hôpital de jour n'est pas un centre de jour, et les patients n'y sont donc que de passage, pour une durée variant généralement entre huit et douze semaines.

Dans un tel contexte, qu' apporte le fait que je sois psychiatre et psychanalyste?

Je dirais d'abord qu'il s'agit de proposer au patient psychotique un dialogue dans lequel pourra éventuellement se déployer quelque chose de sa folie, de sa fracture avec la réalité commune, ce qui est généralement rejeté par ses proches comme des indices de sa maladie. On le sait bien, le psychotique est seul dans l'univers qu'il s'est créé. Si le psychotique investit cet espace de dialogue et qu'il y dévoile sa folie, ce qui est loin d'être toujours le cas, bien sûr il ne s'agit en rien d'interpréter ses symptômes, au sens traditionnel de l'intervention psychanalytique, c'est-à-dire de proposer des liens entre les délires, les hallucinations, le refus d'intégration sociale, d'une part, et, d'autre part, des éléments d'une antériorité inconsciente qui agirait depuis un lieu forclos de sa vie psychique. Dans le 
temps restreint où s'instaure ce dialogue, il s'agit plutôt de donner un lieu de réception possible à la parole du psychotique, qu'il puisse en faire résonner quelque chose pour lui-même et pour l'autre. Et ce dialogue, ce lieu de réception du dire psychotique n'est pas incompatible avec des interventions parallèles qui l'amènent à requestionner de façon cognitive ses convictions psychotiques. Il s'agit là d'un travail très humble qui peut toutefois servir d'amorce à des interventions ultérieures de type psychothérapeutique. Qu'on songe par exemple au dispositif thérapeutique mis en place pour le traitement des psychotiques au 388 à Québec, depuis plus d'un quart de siècle maintenant, et qui place l'intervention psychanalytique au cœur du traitement des psychotiques ${ }^{4}$.

Je dirais ensuite que ce que peut apporter le point de vue psychanalytique dans un dispositif psychiatrique consiste en la prise en compte de la notion qu'il existe une différence structurale qui caractérise le psychotique par rapport au sujet névrotique. Il s'agirait en quelque sorte de convoquer le psychotique vers le lieu d'une subjectivation possible. On le constate souvent en clinique, la subjectivité du psychotique est souvent occupée par ce qui le fait objet de ses hallucinations et de ses délires. Sa propre historicité s'en trouve souvent évacuée. Donc, toute intervention qui l'institue comme sujet de sa propre histoire peut contribuer à introduire un écart vis-à-vis de ce qui le contraint à n'être qu'un objet entre les mains de forces toutes-puissantes. Je considère comme essentiel, notamment, dès les premières semaines de traitement, l'établissement avec le patient de son génogramme, ce qui permet de situer sa place sur le plan inter-générationnel. Bien sûr, devenir un sujet ne va pas de soi, surtout si on se réfère à la notion que tout sujet est un sujet barré, barré d'être assujetti à la loi, à la Loi du langage et de l'Autre. Je fais référence ici à la vaste question du Nom-du-Père et à la façon dont Lacan l'a articulée dans la psychose. Pour cet auteur, le manque de ce signifiant essentiel chez le psychotique conduit à l'instauration de la métaphore délirante qui va faire en sorte que son imaginaire idiosyncrasique recouvrira le champ de la réalité. Je pourrais ici fournir un nombre impressionnant de cas cliniques qui viennent appuyer cette notion d'une carence au niveau de la pierre angulaire du système signifiant (le Nom-du-Père), carence qui est à repérer non seulement dans l'absence ou la défaillance d'un père réel, mais "plutôt dans la place que la mère réserve au Nom-du-Père dans la promotion de la Loi.» (Lacan, 1958). C'est bien autour de cette tentative d'introduction de la loi de l'Autre que se situe au quotidien les interventions proposées au psychotique par les dispositifs psychiatriques, et il n'est certes pas vain d'en tenir compte si on cherche à donner sens à notre travail clinique.

Concernant la question nosologique, le savoir psychanalytique a développé, autour de l'axe odipien, des théories structurales qui orientent encore très habilement nos observations cliniques. La nosographie psychiatrique est une discipline en constante évolution, et ce, depuis les tout débuts de la psychiatrie. Je ne suis pas le seul praticien impliqué actuellement auprès des psychotiques à constater combien de patients déjouent nos classifications et se retrouvent avec des 
diagnostics de psychose brève ou de psychose non spécifiée. C'est-à-dire des patients pour qui le fait psychotique peut n'être qu'itératif ou encore dont les manifestations ne rencontrent aucune catégorisation diagnostique préformée. Le fait de tenter de connaître si ces patients présentent une perte de réalité consécutivement à des mécanismes ressortissant à la névrose ou, plutôt, si cette perte annonce davantage une faille structurale de nature psychotique peut avoir de multiples implications dans l'orientation de nos interventions, bio-psychosociales.

Pour résumer la question des rôles respectifs du psychiatre et du psychanalyste dans mon travail courant auprès des psychotiques, je dirais que la perspective ouverte par la modélisation psychanalytique de la psychose m'amène vers une vision pluridimensionnelle du fait psychotique qui permet notamment d'insérer la production symptomatique dans une perspective plus vaste qui oriente la possibilité du changement psychique escompté dans toute thérapeutique. Dit autrement: tenter de faire parler le symptôme plutôt que de l'éradiquer à tout prix. Puisqu'il ne s'agit en rien de proposer un dispositif psychanalytique à ces patients (qui encore une fois ne sont que de passage pour quelques mois dans le service de l'hôpital de jour lors d'une crise psychotique) et que mon rôle précis au sein de l'équipe interdisciplinaire est celui du psychiatre (avec la responsabilité médicolégale qui y est impliquée), je dirais que ma position d'analyste enrichit mon travail de psychiatre et inscrit celui-ci dans une sorte de parti pris vers l'élaboration symbolique et la croissance psychique.

Sur un plan plus fondamental, il importe que les avancées dans l'un et l'autre champ puissent trouver des zones de correspondance à la mesure de la complexité de l'appareil psychique. Concernant la psychose, prenons par exemple les apports récents de la neuroimagerie cérébrale fonctionnelle. On voit bien que, dans l'évolution de la schizophrénie, certaines zones deviennent hypofonctionnelles ou atrophiées, comme les lobes frontaux ou les hippocampes, respectivement reliées à des fonctions cognitives évoluées et à la mémoire ou au stockage d'informations. Or ces données peuvent être corrélées à ce qui s'élabore du côté de la psychanalyse concernant les psychoses, notamment avec cette faille dans le procès de la symbolisation qui rend si prévalent l'imaginaire du psychotique à s'emparer de la réalité. Il est tout à fait intéressant de faire parler ces données probantes dans l'éclairage de ce qui est différent chez le psychotique dans son appareil à penser et dans la formalisation de son discours, de son rapport à l'autre. Autrement dit, pour donner sens à ce que les données de l'imagerie apportent, l'activité spéculative de la psychanalyse, dans ses liens notamment avec la linguistique et la philosophie, peut donner lieu à un dialogue tout à fait créatif.

Par ailleurs, il est bien évident que les psychotiques savent parler et que, si on veut approcher la singularité de leur rapport au langage qui se fait jour à travers notamment le délire et les hallucinations auditives, il faut pouvoir remonter à la racine du processus par lequel le langage est intégré à la vie psychique. Cela rejoint les champs de recherches de certains cognitivistes qui se rendent compte 
que le mot - la dimension phonétique du mot — est inter relié à toute une cascade de fonctions cérébrales responsables d'articuler l'individu non seulement avec son environnement, mais également avec ses processus mnésiques et oniriques. Ce qui n'est pas si éloigné de ce que Lacan n'a cessé d'élaborer, à savoir que c'est le signifiant qui entraîne à sa suite la signification, et non l'inverse, et donc que le signifiant n'est pas le simple valet de la signification avec, au-delà, la réalité qui attendrait d'être expliquée.

À l'ère de la plasticité neuronale, les avenues ne manquent pas pour dynamiser les hypothèses par lesquelles les aléas développementaux peuvent entraîner des modifications dans certaines structures cérébrales, aléas qui auront des répercussions sur le plan psychopathologique. Mais dans la mesure où l'on comprend que certaines de ces modifications sont réversibles, il devient primordial de mettre en place des modèles de compréhension et d'intervention qui sont à la mesure de la complexité de l'objet étudié.

\section{Un dialogue encore possible?}

Arrivant au bout de cette réflexion, une question se pose avec acuité: le dialogue est-il encore possible entre psychiatrie et psychanalyse ?

Au point où en sont les choses, il est tout à fait pensable qu'en un avenir rapproché la psychanalyse ne fasse plus partie que de l'histoire de la psychiatrie et qu'elle soit disparue de la formation des futurs psychiatres, du moins en Amérique du Nord. En effet, considérant le processus de financement de la recherche et de la formation médicale continue, la sélection des futurs professeurs dans les départements de psychiatrie, l'obsession de ne pouvoir approcher la vérité qu'à partir de données probantes, avec en toile de fond le fantasme prévalent d'une machine humaine délivrée de toutes ses ombres inconscientes et parfaitement adaptée à son environnement, tout est en place pour la venue de cette éventualité.

Mais comment ne pas voir alors qu'il s'agirait d'un appauvrissement majeur du champ même de la psychiatrie, d'une restriction de son regard ? Et quel discours scientifique pourra-t-il prendre le relais en vue d'une compréhension structurale de l'intra psychique?

Du côté de la psychanalyse, il importe également qu'elle soit soucieuse du renouvellement de son discours dans l'espace du savoir actuel, sans se cantonner dans des enjeux de frontière où elle s'est souvent isolée et appauvrie, voire même coupée de ses concepts fondamentaux. Plus occupée à définir qui est psychanalyste et qui ne l'est pas qu'à transmettre un savoir vivant dont la valeur peut sans cesse féconder et rouvrir notre regard clinique. En ce sens, il devient urgent que la psychanalyse reprenne sa place dans le champ de la formation psychiatrique continue et qu'elle soit plus hardie à sortir hors de ses murs afin de se risquer dans l'espace du dialogue. Transmettre les rudiments psychodynamiques est évidemment essentiel dans le curriculum de la résidence en psychiatrie, mais qu'est-ce qui s'offre ensuite au psychiatre pour guider son questionnement dans la complexité changeante du fait clinique? 
Nous nous trouvons présentement à une sorte de carrefour où ce qui se joue entre psychiatrie et psychanalyse se déploie sur un fond plus global des mutations de la subjectivité. Avec l'ouverture sans précédent des méthodes de communication, la prolifération de l'information, l'extension du virtuel jusque dans les modes d'apprentissage des jeunes enfants, le métissage des cultures, il est bien évident que le sujet post-moderne s'engage dans des voies qui deviennent tout à fait imprévisibles. Est-ce que la psychiatrie va contribuer au processus de désubjectivation en alignant la souffrance psychique sur le paradigme d'une tare objectivable à éradiquer ou va-t-elle plutôt tenter de préserver ce qui la spécifie au sein du grand ensemble médical?

C'est bien ici que, revenant à Barthes, il s'agit de faire jouer la question de la différence en tant qu'elle se conquiert sur le conflit. Si on doit jouer la lettre contre le chiffre, c'est en effet, d'une part, selon le principe d'un antagonisme entre le pouvoir qui se dégage de la singularité recélée à l'intérieur de tout dialogue thérapeutique et, d'autre part, la force des vérités qui sont probantes d'être quantifiables et réitérables. Nous l'avons vu, cet antagonisme est bien réel et peut conduire à des exclusions péremptoires de part et d'autre. Mais il importe de ne pas se laisser piéger dans cet antagonisme et d'aller au-delà et à côté de lui pour saisir les véritables enjeux. On s'aperçoit alors que les frontières entre la psychiatrie et la psychanalyse sont poreuses et qu'au-delà du conflit, la prise en compte des différences mène à des rapports qui peuvent féconder et le savoir et la pratique de l'une et l'autre disciplines. Le véritable défi se tient dans la particule élémentaire entre psychanalyse et psychiatrie, en tant qu'elle indique à la fois leur séparation et leur proximité, leur différence radicale mais certainement pas irréconciliable.

Je n'ai pas développé toute cette argumentation et ce détour par l'épistémologie dans le dessein nostalgique que la psychanalyse reprenne la place qu'elle a eue pendant quelques décennies au sein du corpus de la psychiatrie. Les enjeux actuels m'amènent plutôt à prôner d'abord la mise à jour d'un véritable débat de fond entre les différences qui caractérisent l'un et l'autre domaine, soit sortir de ces disqualifications souvent puériles et «trendy » qui s'expriment dans le discours de nombre de psychiatres envers la psychanalyse ou qui, sous le couvert d'efficacités administratives, balayent du revers de la main la question du sens (des symptômes, de leurs actions, de la signification du psychopathologique dans les changements du macrocosme social, etc.). Ensuite, parce que je crois qu'en tant que science de l'inconscient, la psychanalyse peut très bien évoluer au-delà et à côté de la psychiatrie, en marge certes, mais depuis un lieu incontournable - un lieu autre qui travaille à sa façon, à partir du pouvoir de la lettre et du spéculatif, l'assurance de notre savoir, qui ne sera toujours qu'un conglomérat de vérités minuscules.

gilles chagnon

hôpital maisonneuve-rosement montréal (québec) h1t 2 h1

gilles.chagnon@videotron.ca 
Filigrane, automne 2007

\section{Notes}

1. Je fais référence ici à l'étymologie du mot même de psychiatrie: soigner l'âme.

2. «Recommandation 22: Individual and group psychotherapies adhering to a psychodynamic model (defined as therapies that use interpretation of unconscious material and focus on transference and regression) should not be used in the treatment of persons with schizophrenia. »

3. L'équipe se compose, outre le psychiatre et le résident, de deux infirmières, d'une ergothérapeute, d'une travailleuse sociale.

4. Je fais référence ici aux recherches et au travail considérable de l'ABC du GIFRIC, soit Willie Apollon, Danielle Bergeron et Lucie Cantin, ainsi qu'à tous leurs collaborateurs. Cela représente une illustration très probante de l'apport de la psychanalyse dans le traitement de la psychose.

\section{Références}

Ansermet, F., Magistretti, P., 2004, À chacun son cerveau, Paris, Odile Jacob.

Apollon, W., Bergeron, D., Cantin, L., 1990, Traiter la psychose, Gifric.

Assoun, P.-L., 1981, Introduction à l'épistémologie freudienne, Paris, Payot.

Assoun, P.-L., 1984, 3, Paris, Gallimard.

Barthes, R., 1973, Le plaisir du texte, Paris, Seuil.

Bergeron, D., 2004, Un parti pris pour la psychanalyse, Santé mentale au Québec, in Rencontres avec des pionnières en santé mentale.

Bergeron, D., 2005, Les enjeux de la psychiatrie actuelle et son avenir. Pour une psychiatrie clinique éthique, Santé mentale au Québec, Où va la psychiatrie?, vol. XXX, n 1, 197-214

Cantin, L., 2005, L'évaluation «objective» des traitements en santé mentale ou quand la recherche clinique est réduite à la méthode expérimentale, Santé mentale au Québec, Où va la psychiatrie ?, vol. XXX, nº 1, 183-196

Chomsky, N., 1979, Théorie du langage et théorie de l'apprentissage, Paris, Seuil.

Derrida, J., 1967, De la grammatologie, Paris, Éd. de Minuit.

Derrida, J., 1997, Résistances — de la psychanalyse, Paris, Galilée.

Derrida, J., Roudinesco, E., 2001, De quoi demain..., Paris, Flammarion.

Freud, S., 1919, Faut-il enseigner la psychanalyse à l'université ?, in Euvres complètes, tome XV, Paris, PUF.

Freud, S., 1924, Névrose et psychose, in Névrose, psychose et perversion, Paris, PUF.

Freud, S., 1924, La perte de réalité dans la névrose et dans la psychose, in Névrose, psychose et perversion, Paris, PUF.

Kristeva, J., 1981, Le langage, cet inconnu, Paris, Seuil.

Kolko, C., 2000, Les absents de la mémoire, Figures de l'impensé, Paris, Érès.

Lacan, J., 1955-56, Séminaire III. Les structures freudiennes des psychoses, Paris, Seuil.

Lacan, J., 1958, D’une question préliminaire à tout traitement possible de la psychose, in Écrits, Paris, Seuil.

Lacan, J., 1971-72, Le savoir du psychanalyste, in L'association freudienne internationale, 1971-72.

Lehman, A., Steinwachs, D., 1998, Translating Research Into Practice: The Schizophrenia Patient Outcomes Research Team (PORT) Treatment Recommandations, Schizophrenia Bulletin, vol. 24, $\mathrm{n}^{\circ} 1$.

Meyer, C. et coll., 2005, Le livre noir de la psychanalyse, Les Arènes.

Michaud, Y., 2002, Le cerveau, le langage, le sens, Paris, Odile Jacob. 
Peraldi, F., Le sujet, Séminaire 1981-82, Voix psychanalytiques, Montréal, Liber.

Peraldi, F., 1978, L'élangage de la folie, Santé mentale au Québec, vol. III, nº 1, 1-17

Pommier, G., 2004, Comment les neurosciences démontrent la psychanalyse, Paris, Flammarion.

Ver Eecke, Wilfried, 2003, The Role of Psychoanalytic Theory and Practice in Understanding and Treating Schizophrenia: A Rejoinder to the PORT Report's Condemnation of Psychoanalysis, Journal of The American Academy of Psychoanalysis and Dynamic Psychiatry, vol. 31, $\mathrm{n}^{\circ} 1$. 